\title{
Community Psychiatric Nursing
}

\section{$A$ Discussion Document by a Working Party of the Social and Community Psychiatry Section*}

Introdnetion.

Community psychiatric nursing has developed rapidly in recent years and there is a great variation between services and in operational policies. It seemed appropriate that there should be some discussion within the College as to the role of the community psychiatric nurse, lines of responsibility and communication and relationship with other professional groups.

The present Working Party was set up by the Social and Community Psychiatry Group in 1978. Representatives of the Community Psychiatric Nurses Association and British Association of Social Workers attended some of the meetings. Written comments were received from the Community Psychiatric Nurses Association, British Association of Social Workers, British Psychological Society, Health Visitors Association, Council for Education and Training of Health Visitors and the Royal College of Nursing. Letters were received from the Child and Adolescent Psychiatry and the Forensic Psychiatry Sections.

There was agreement on most aspects of the work of the community psychiatric nurses, but some differences of opinion on certain points amongst some of the professional groups who were consulted, including comments on a number of difficult problems concerning roles and responsibilities on which we concentrate in this report.

Development of community paychintric nuraing.

The development of community psychiatric nursing is part of the changing pattern of psychiatric care moving from the isolated, self-sufficient asylum to the non-custodial care in the community. Psychiatric nursing developed within the mental hospitals and was influenced by ideas ranging from 'moral treatment' to the enthusiasm for somatic investigation and treatment of the early 20th century. In recent years the range of therapies carried out by nurses within the hospitals has widened and the ratio of staff to patients has increased. With the development of early discharge policies it was logical that the nurses who understood the needs of psychotic patients should follow them in the community. At first it was a minor aspect of ward-based nurses' work, but later nurses with a primary commitment to community work were appointed. This allowed the development of special skills, but made it more difficult to use the important links that had been forged between the patient and the psychiatric unit.

- Members of the Working Party: Drs A. C. Brown (Convenor); D. Bennett; J. D. W. Fisher; P. Jefferys; Profs. I. Marks; E. Paykel; Drs M. Richards (co-opted: RCGP); Diana Dickens; C. P. Seager; C. M. Parkes; Mr P. Mellor (co-opted: RCN); Dr M. Y. Ekdawi; Mr P. Carr (co-opted: RCN).
Within recent years the concept of the multidisciplinary team approach to psychiatric care has been widely accepted. As a member of the team the community psychiatric nurse is able to meet patients on the ward and attend team meetings, but spends most time visiting patients at home, in hostels or elsewhere in the community. Psychiatric nurses working in the community make contact with other community workers, such as general practitioners, health visitors, district nurses and social workers and see patients who have never been in hospital. After-care remains important but increasingly community psychiatric nursing forms part of a district service which is less hospital-orientated.

\section{Present roles of community psychiatric nuree.}

There is great variation between one service and another as to the role of the community psychiatric nurse. Inevitably it involves facets of other professional activities and it is therefore important to try and define clearly those areas which are specifically nursing and those where there is overlap. Liaison is important to ensure that two people are not doing the same work with the same individual or family. Firstly, there are direct services to the patient. These include visiting former in-patients and their families and offering long-term support. There is the identification of specific problems and the referral to appropriate resources. The nurse also has the responsibility of assessing former patients for evidence of change, particularly deterioration which may require further attention, and the nursing assessment at home of newly referred patients. There is the administration of medication, particularly long acting antipsychotics and the assessment of their effect. Some CPNs also undertake behavioural psychotherapy. A certain amount of physical nursing, especially with elderly patients, may be needed. In addition to visiting patients at home, work in out-patient clinics, after-care hostels, day centres and other nonpsychiatric community resources may be important. Recent studies, such as those of Sladden (1979) and Tough et al (1980), have described the time spent on different types of work.

The second function is to act as liaison between the hospital service and the primary health team, in particular the general practitioner. The nurse, therfore, may be expected to attend meetings held at Health Centres with members of the primary team as well as those of the psychiatric team. Similarly, the psychiatric nurse may act as adviser to other nursing colleagues, such as district nurses, health visitors, accident and emergency centres and similar groups. Contact with Social Services is also important.

Other activities may include visiting 'at risk' groups, 
including persons who have previously taken overdoses of drugs, parents of non-accidentally injured children, battered wives, alcoholics, gamblers or others with psychiatric problems who may come to their attention, not necessarily through the medical services but possibly through social services or the police. In undertaking these tasks the work of the CPN overlaps greatly with that of social workers and health visitors.

\section{Relationship with the psychiatric unit.}

It was agreed that close links with a consultant psychiatrist and other members of the multidisciplinary psychiatric team were essential. The extent to which staff involved in community psychiatric nursing should be separate from those engaged in in-patient and day-patient care was questioned. Opinions within the Working Party and outside ranged from the view that all nurses in a district psychiatric unit should follow up patients in the community to seeing community psychiatric nursing as a separate job with its own career structure.

The advantages of all nurses going into the community are continuity of patient care and good communications within the team. The problems of this approach are conflict of responsibility between the needs of patients in the ward and patients in the community and the need for special training for community work. There are also administrative difficulties concerning transport, lines of responsibility and conditions of work. It was agreed that the community psychiatric nurse should be a fully integrated member of the multidisciplinary psychiatric team and should attend all essential team meetings. On balance it was accepted at this stage that some nurses have to be appointed specifically for community work, but that other nurses in the psychiatric team could do some follow-up visits and pre-admission assessments in the community.

Whatever the specific arrangements, we regard the most satisfactory pattern of working as one in which the individual nurse works in relationship to a single psychiatric multidisciplinary team and referrals are handled directly within the team.

\section{Relations with the primary care team.}

General practitioners have on the whole welcomed the development of community psychiatric nursing services, but with some reservations. When the CPN is not part of the primary care team her independent operation may conflict with the GP's aims. It was agreed that communication with the primary care team is essential. Many general practitioners would like CPN's to be health centre based and so be members of the primary care team. This has implications for manpower requirements which will be discussed later. Communication is obviously simplified if the CPN has only to relate to a small number of GPs in a defined area.

We would suggest that at this stage in the development of services the CPN should be a member of the psychiatric team but should keep closely in touch with the primary health care workers and social workers in the community. Referrals by GPs and their colleagues to the CPN should be viewed as referrals to the psychiatric team and should be discussed in team meetings. When CPNs are a limited resource and demand is great, referrals may be best screened by the consultant psychiatrist, but where pressures are not so great direct referrals from GPs should be welcome.

The move of specialist services out of hospital into the community may raise problems concerning clinical responsibility. In particular it is important to determine whether the consultant psychiatrist or the GP is controlling the prescription of drugs given to patients cared for by CPNs. Once again, good communication is the key to success. The ease with which the community psychiatric nurse can work along with the primary care team may in the end be a measure of how well the psychiatric team is integrated with the community it serves.

\section{Relationship with other professions.}

In addition to the relationship between CPNs and the established members of the primary health care team, we considered the role overlaps and relationships with a number of other groups.

Many general practitioners have seen the CPN as taking over some of the work formerly done by Mental Welfare Officers. At the same time community-based social workers have taken on some mental welfare tasks, but have to give priority to statutory responsibilities, particularly in the field of child care. There is need for a continuing discussion as to the areas of responsibility of CPNs and social workers. Where social workers and CPNs are part of the psychiatric team a reasonable sharing of work should present no problem.

In recent years there has been some development of community work by other staff based at psychiatric hospitals and units. Clinical psychologists have been expanding their role and in some areas work with primary care teams. Occupational therapists and other remedial therapists would like to do more domiciliary work. Such developments are needed if psychiatric services are to become increasingly community-orientated. Role boundaries will inevitably, and often usefully, be blurred, so good communications between professional groups in the community will be essential.

The home help service plays a vital role in maintaining many disabled people in the community. CPNs can offer valuable support to home helps, particularly in the care of the elderly. There is some overlap between the tasks done by CPNs and those done by home helps, particularly in caring for elderly people living alone. In some areas the introduction of specialist home helps has increased the efiectiveness of community care.

The staff of day centres and welfare homes for the elderly 
may also tenefit from support from CPNs. Members of voluntary organizations may need help in their work with disturbed people and the CPN may be the best placed member of the psychiatric team for them to contact.

\section{Specialization.}

We had considerable discussion as to the degree of specialization that is desirable in community psychiatric nursing. Certain areas in particular lend themselves to specialization.

Mental Handicap: The need for specialist mental handicap community nurses was generally agreed, as the nurse in mental handicap has a different qualification. Fewer mental handicap patients require longer term nursing support in the community than do mentally ill and elderly patients. The community psychiatric nurse (mental handicap) should be a member of the community mental handicap team and related to the consultant and the specialist social worker. Attachment to primary health care teams is not feasible in view of the small numbers of patients involved, although the CPN should liaise both with the specialist team and with the GP and his colleagues.

Psychiatry of Old Age: One major emphasis in old age psychiatry is the support of patients and their relatives at home in the community or in sheltered accomodation, so avoiding or postponing long-term hospital admission. Not surprisingly, specialist community psychiatric nursing has developed, and a strong case was made for a group of nurses to work with each psychiatric team. As always, relations with nurses and health visitors were seen as important and there may be difficulties in defining the respective roles of the district nurse. We would recommend that where there is a specialist psychiatric team this should include a group of specialist nurses.

Child Psychiatry: Child psychiatry has developed independently from general adult psychiatry and has its own special therapeutic skills and style. There is therefore a case to be made for specialist community nurses working with children and adolescents. So far there are very few such services developed. The number of specialist nurses would be relatively few and they certainly would be unable to integrate with primary care teams, but would, of course, liaise closely with them. The work of the specialist community nurse in child psychiatry would overlap greatly with that of the social worker and health visitor, and their respective roles need evaluation.

Forensic Psychiatry: With the development of specialist forensic psychiatric units serving health areas or even having a regional or sub-regional catchment area, the careful supervision of discharged patients will be most important. It has been suggested by the Forensic Psychiatry Section that some of the follow-up of patients discharged from specialist units should be done by nurses attached to these units. Although this was agreed by the Working Party, it raises, once again, the respective roles of nurses and social workers.

Behavioural Psychotherapy: Much behavioural work is already carried out by general CPNs. A specialist CPN is the nurse-therapist whose main role is to give brief behavioural psychotherapy for neurotic patients, usually in out-patient settings, but commonly also in the home and recently in primary care. Though much of their work is in the community, these nurses go through a separate JBCNS course (No. 650) which involves in-service training and instruction in evaluation of patient outcome JBCNS qualification (Courses No. 800 and 650). Modification of the courses is desirable in that they overlap and the overall training time could be reduced.

Other Specialist Services: Crisis intervention, rehabilitation, alcoholism and addiction have also been seen as areas for specialist community psychiatric nursing. The inclusion of CPNs in crisis assessment teams is still being explored. The CPN can provide continuity of care and a link between in-patient, out-patient and primary care services. Further work is needed to determine the circumstances in which the CPN would assist the social worker and/or psychiatrist in carrying out crisis assessments and short-term family therapy.

In this field of work, as in others, there is the tendency for services to be started using whatever professional manpower is available or already working together in another setting. It raises the issue of the best basic training for community mental health workers-medical, nursing, psychology, social work? In this work personality and experience are so obviously important, but the appropriateness of professional education and training is equally so.

Where there is a separate CPN service for old age psychiatry, an increased proportion of the work of the other CPNs working with adults will inevitably concern those receiving long-term support and rehabilitation. However, any specialist rehabilitation unit might make a reasonable case for having its own community nurse who understands the particular aims of the programmes developed for individual patients.

Alcoholism/addiction units may, like other specialist units, make a case for specialist CPNs or for sending unit staff to visit patients at home.

\section{Training and career structure.}

The majority of community psychiatric nurses at the present time have not attended formal training courses but have had some type of in-service training. According to evidence given by the Community Psychiatric Nurses Association, in September 1978 only 10-15 per cent had completed the Joint Board of Clinical Nursing Studies Courses 800 and 989. A similar number had completed 
management-orientated community psychiatric nursing courses (such as the NEBSS course). With respect to specialized CPNs there are also 60 nurse-therapists trained or training in the JBCNS Course No 650 .

There is need for review of suitable training courses for those entering CP nursing. The development of shorter courses in addition to the present courses both for new entrants and for established but untrained CPNs is welcomed. In particular, there is a need for a short course for experienced CPNs or a day release type of course. If working in the community is not to become unduly separated from the rest of psychiatric nursing, all psychiatric nurses should be given an expanded period of community training. The basic nurse training should become increasingly community-orientated, so that the newly qualified psychiatric nurse can move as easily to community work as to work with in-patients or day patients.

As with other branches of nursing, it is essential that community psychiatric nursing should develop a career structure that allows those with the interest, training and experience to remain in clinical nursing rather than have to move into administrative roles to gain promotion and increased salaries. Interchange between hospital and community work should be encouraged, but it should also be possible for relatively senior nurses to find appropriate positions for continuing their clinical work in the community.

\section{Manpower implications.}

As community psychiatric nursing services develop, new areas of work for nurses become apparent. At present there is an overall ratio of about one CPN to every 40,000 population, although the distribution of community psychiatric nursing services throughout the country is extremely variable. Brook and Cooper (1973) suggested a ratio of one CPN to every 30,000 population. Those who want to see CPNs closely associated with primary health care teams suggest a ratio of one CPN to every $12-15,000$ population, which means one CPN for every 5-6 general practitioners and every three district nurses. The Community Psychiatric Nurses Association suggest a ratio of one CPN to every 7,500 population. Before planning expansion on this scale, the respective roles of the community psychiatric nurse, district health visitor and social worker have to be considered, and also the relative numbers of generalist and specialist community psychiatric nurses. If it were hoped to develop a nationwide service of CPNs attached to primary care teams, the members of CPNs would have to be at least trebled to meet this need alone. If in addition specialist units and services were to have their own CPNs, the manpower increase would have to be four or five fold. Evaluation studies of existing services or specially set up pilot schemes are needed to determine whether such increases in manpower are justified in terms of reducing the work of existing services or improving the quality of patient care. The majority of patients with psychiatric problems, including a significant proportion of those with serious chronic disorders, are managed by the primary care team without any contact with the psychiatric team. The aim of specialist services must be to provide support for this work.

As mentioned above, a fundamental problem is to determine the best training for basic mental health care workers in the community. In hospital work nurses still outnumber all other professional staff and provide the basic continuing care of in-patients and day patients in most situations. With the increasing and continuing trend to community care for the mentally ill, there is a strong case for seeing community psychiatric nurses as providing continuity of specialist care. This implies a considerable expansion in numbers of CPNs possibly to around that suggested by the Community Psychiatric Nurses Association. This would also need changes in basic nurse training.

There is a great need for experimentation with different styles of service at this stage. We would urge the DHSS to support experimental schemes and to avoid any attempt to standardize the pattern of services until the various different styles have been evaluated. Care should also be paid to greater training of CPNs in evaluation of the outcome of their interventions with patients.

There may also be scope for employing nursing assistants in the community working under the supervision of trained CPN's, particularly with chronically ill and elderly patients. Wherever possible, trained staff should be allowed to devote their time to those tasks for which their training is really needed. Untrained staff should be taken on to do some of the work which is similar to that done by staff of welfare homes or by family members when they are available.

\section{Financial implications.}

At a time of financial constraint, any expansion of service has to be achieved by reducing costs elsewhere. If CPN services are to be developed, this implies giving them considerable priority over most other aspects of mental health care. In the long term it may be reasonable to hope that developing community services will allow for some reduction in more expensive hospital facilities, and so there will be some saving, but at present it would be unreasonable to make any major reduction in existing services to develop alternatives. Expansion of CPN services may therefore have to be slower over the next few years but should proceed wherever possible.

\section{Research and evaluation.}

Many descriptive studies and a smaller number of evaluative studies have already been published. The literature on community psychiatric nursing has been reviewed by Carr (1980), and by Griffiths and Mangan (1980). Since the first evaluation studies, such as that of Hunter (1974) were carried out, practice has changed and will no doubt continue 
to change. There is therefore a need for continuing evaluation. Comparisons should be made, not only between CPN services and other approaches but between different styles of CPN services. Evaluation should include not only reasons for clinical and social improvement but also assessment of cost effectiveness.

\section{Summary and recommendations.}

We have considered the development and present roles of community psychiatric nursing services and have come to the following conclusions:-

1. The development of community psychiatric nursing services has been of benefit to patients, has assisted in the development of community psychiatry and may have reduced pressure on hospital resources. These services should be maintained and expanded. This expansion should be monitored to ensure that the expected benefits can be demonstrated.

2. There is a need to consider the development of 24-hour, seven days a week CPN services. Evaluation of such developments is important to show whether the increased commitment is justified.

3. At present the CPN should be a member of the psychiatric team, but in the future if numbers increase it may be possible for CPNs also to be members of primary care teams. The value of CPNs working as members of primary care teams requires evaluation.

4. There is need for further experimentation and no one pattern of CPN service can at this stage be seen as ideal.
5. The training of psychiatric nurses should become increasingly community-orientated. Shorter courses should be made available so that all CPNs have some formal training.

6. Psychiatric nurses working with in-patients and day patients should have the opportunity to follow up patients in the community when continuity of care or other factors make this desirable.

7. The expansion of CPN services should not be so rapid that it depletes hospital in-patient services of staff and so enciangers the care of the chronically ill.

8. There should be a degree of specialization in CPN work and evaluation studies should be made comparing 'specialist' and 'generic' services.

\section{REFERENCES}

Carr, P. J., Butterworth, C. A. and Hodges, B. E. (1980). Community Psychiatric Nursing. Edinburgh: Churchill Livingstone.

Grifftr, J. H. and MAngen, S. P. (1980). Community peychiatric nursing: A literature review. International Journal of Nursing Studies. (In press).

HUNTER, P. (1974). Community psychiatric nursing in Britain: An historical review. International Journal of Nursing Studies, 2, 223-33.

Sladden, S. (1979). Psychiatric Nursing in the Community. A Study of a Working Situation. Edinburgh: Churchill Livingstone.

Tough, H., Kingerlee, P. and Eulotr, P. (1980). Surgetyattached psychogeriatric nurses: an evaluation of psychiatric nurses in the primary care team. Journal of the Royal College of General Practitioners, 30, 85-89.

\title{
Trainees' Forum
}

Contributions are welcome from trainees on any aspects of their training

\section{A View on Senior Registrar Training in General Adult Psychiatry}

\author{
By Robert Fieldsend, Senior Registrar, Warneford Hospital, Oxford
}

Pre-Membership training tends to concentrate on the acquisition of knowledge and gaining clinical experience, especially in the assessment of patients. In postgraduate training the learning of various management skills, the development of special interests and filling gaps in previous training are the primary tasks. Theoretical learning through books and journals alone is insufficient in these areas. Opportunities must be available for the senior registrar to use his clinical time to achieve satisfactory training. If the senior registrar's position is largely supernumerary this is easily achieved; however, this happy situation is a rarity.

In preparing for consultantship, the higher trainee needs to develop team management and administrative skills, become accustomed to working in a supervisory capacity, increase his teaching experience, and be able to initiate and carry through changes in the institution or service he is providing. Most clinical placements of over one year should be able to give this experience; acting for a consultant on leave is not adequate training. As well as receiving advice and guidance the trainee needs the opportunity to have a reasonably free hand in a particular area, e.g. altering a ward culture, running a liaison service, setting up supervision and training for particular staff members. It is possible that in a non-teaching hospital there may be less constraint on such activities. In practice it is important that the consultant should be flexible and interested in helping the traince with these skills 